\title{
Importancia de Melophagus ovinus como vector de enfermedades en varias partes del mundo
}

\author{
Casco, X.; Roldán, J.; Serrano, D.; Simbaña, M.; Soria, C. \\ Universidad Central del Ecuador. acsoria@uce.edu.ec; ljroldan $a$ uce.edu.ec; \\ masimbanaj $@$ uce.edu.ec; cxcasco@uce.edu.ec; dfserrano@uce.edu.ec
}

\begin{abstract}
Resumen
Casco, X.; Roldán, J.; Serrano, D.; Simbaña, M.; Soria, C.: Importancia de Melophagus ovinus como vector de enfermedades en varias partes del mundo. Rev. Vet. $32: 1, X X-X X$, 2021. Melophagus ovinus es un parásito hematófago distribuido a nivel mundial. que tiene una actuación fundamental como vector, por facilitar la entrada de agentes patógenos a los animales. Se realizó una búsqueda de artículos en la base de datos de Pubmed relacionados con el tema a investigar. El objetivo de esta revisión se basa en establecer la importancia del parásito como transmisor de enfermedades y describir el efecto patógeno en el ámbito veterinario, así como enumerar los principales agentes relacionados a $M$. ovinus tales como Trypanosoma melophagium, Anaplasma sp, Rickettsia sp, Bartonella melophagi, Theileria $s p$, virus de la lengua azul y Borrelia burgdorferi. así como los efectos generados en la salud animal. En conclusión, se estableció la importancia de $M$. ovinus como vector de enfermedades en varios países del mundo (Australia. China. India. Etiopia. Canadá. Estados Unidos. países del Medio Oriente y América del Sur) y de esa manera. el futuro efecto zoonótico de los patógenos relacionados con $M$. ovinus y su impacto en la producción animal.
\end{abstract}

Palabras clave: Melophagusovinus, agentes patógenos. pubmed. vector.

\begin{abstract}
Casco, X.; Roldán, J.; Serrano, D.; Simbaña, M.; Soria, C.: Importance of Melophagus ovinus as a disease vector in various parts of the world. Rev. Vet. 32: 1, XX-XX, 2021. Melophagus ovinus is a hematophagous parasite distributed worldwide, which plays a fundamental role as a vector. facilitating the entry of pathogens into animals. A search was carried out for articles in the Pubmed database. which are related to the topic to be investigated, in which 81 articles were analyzed. and 10 of them were taken that focused on agents that we consider most important in M. ovinus, therefore, the objective of this review is based on establishing the importance of this parasite as a transmitter of diseases globally, describing the pathogenic effect in the veterinary field. and finally, listing the main agents related to $M$. ovinus. such as Trypanosoma melophagium, Anaplasma sp, Rickettsia sp, Bartonella melophagi, Theileria $s p$, bluetongue virus and Borrelia burgdorferi, as well as the effects on animal health. In conclusion, the importance of $M$. ovinus as a vector of diseases was established in several countries of the world (Australia. China. India. Ethiopia. Canada. United States, countries of the Middle East and South America). and thus, the future zoonotic effect of pathogens related to $M$. ovinus and their impact on animal production.
\end{abstract}

Keywords: Melophagus ovinus, pathogens, pubmed, vector.

\section{INTRODUCCIÓN}

Melophagus ovinus (piojo o garrapata de la oveja) es una forma de vida parásita, hallada principalmente en ovinos ${ }^{12}$. En su forma adulta es de color pardo oscuro. con estructuras para aferrarse a su hospedador. Posee una forma aplanada y se define como un insecto hematófago perteneciente a la categoría de los dípteros en la familia Hippoboscidae ${ }^{2}$.

Este agente se puede hallar en las regiones donde existan bajas temperaturas ${ }^{13}$. Dependiendo de la tem-

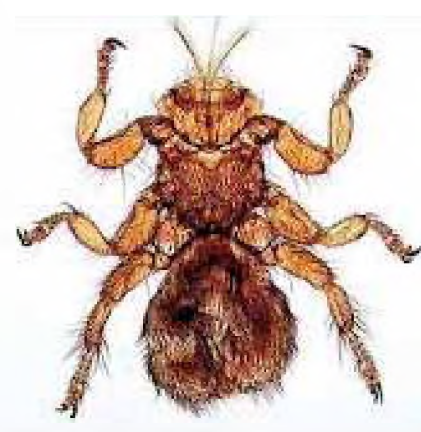

Melophagus ovinus 
peratura ambiental, el parásito tendrá las condiciones para reproducirse y completar su ciclo vital ${ }^{2}$.

M. ovinus posee un ciclo biológico de tipo directo que dura un mes aproximadamente, en el cual el huevo necesita madurar para convertirse en larva. Este proceso de maduración se realiza en las hembras parásitas a nivel abdominal, luego de que se produjo la maduración del huevo.

La larva resultante se transforma en crisálida, la cual en períodos de una a dos semanas, se convierte en pupa, y es en esta fase donde el parásito se ubica cerca de la piel donde evoluciona hasta convertirse en adulto ${ }^{14}$

La enfermedad que produce este parásito es descrita como melofagosis, la cual produce en el animal intensa picazón, pequeñas úlceras a nivel de piel, estrés e irritación debido a la acción del parásito, como también por la presencia de tierra y agentes contaminantes en las heridas producidas ${ }^{1}$.

M. ovinus tiene una distribución a nivel mundial ${ }^{1}$. Debido a que es un parásito hematófago lesiona la piel, facilitando la entrada de ciertas bacterias, protozoarios y agentes patógenos, los cuales ayudan a generar una herida con mayor grado de afectación hasta formar úlceras. Además, es considerado vector de ciertos protozoarios $^{8}$.

En este estudio se recopilaron varios documentos con información acerca de algunas enfermedades encontradas en el parásito, que destacan la importancia de reconocerlo como un vector de consideración en veterinaria y en salud pública.

Los objetivos de este trabajo fueron establecer la importancia de $M$. ovinus como vector de enfermedades en varias partes del mundo, describir el efecto patógeno del parásito dentro del ámbito veterinario y enumerar los principales agentes patógenos de $M$. ovinus y sus efectos en la salud animal.

\section{MATERIAL Y MÉTODOS}

Para la presente revisión se realizó una estrategia de búsqueda basada en la base de datos de Pubmed, donde se indagaron artículos relacionados con el tema, sin tomar en cuenta la fecha del estudio.

Para ello se usaron las palabras clave "Melophagus ovinus", dando como resultado 81 artículos, de los cuales se seleccionaron 20 papers mediante la lectura de los temas y los resúmenes de cada uno.

Se excluyeron los que no eran relevantes al tema de enfermedades de $M$. ovinus. En cambio se seleccionaron los agentes considerados más importantes (Trypanosoma melophagium, Anaplasma sp, Rickettsia sp, Bartonella sp, Theileria sp, virus de la lengua azul y Borrelia burgdorferi). Se tuvieron en cuenta los documentos que trataban a fondo el tema, obteniéndose en total 10 artículos para los resultados de la revisión.

\section{RESULTADOS}

\section{Trypanosoma melophagium}

La oveja ked Melophagus ovinus se conoce como el ectoparásito de ovejas erradicado del Reino Unido debido al uso de plaguicidas, sin embargo persiste en la oveja salvaje Soay de StKilda en las Hébridas Exteriores, frente a las costas de Escocia. Los keds de oveja transmiten Trypanosoma melophagium, pero no ha sido registrado en la oveja StKilda. Se realizaron pruebas de PCR para detectar los tripanosomas en keds conservados y en frotis de intestino. La mayoría de los keds poseen tripanosomas, recolectados de corderos ${ }^{15}$.

\section{Anaplasma}

En relación a la anasplasmosis con vector $M$. ovi$n u s$, en un estudio realizado en Xinjiang del Sur (provincia china), alrededor de 93 especímenes (incluidos ocho especímenes de pupas de $M$. ovinus), se seleccionaron para el aislamiento de ADN genómico, PCR y secuenciación del gen msp4 de Anaplasma sp. En 2013, los resultados arrojaron que las muestras estaban libres de Anaplasma sp, mientras que en 2016 y 2017 salió positivo a esta bacteria. El análisis de 24 secuencias del gen msp4 (de cuatro especímenes de pupas) confirmó la existencia de $A$. ovis en especímenes de $M$. ovinus recolectados en China. Con este estudio se confirma la transmisión vertical de $A$. ovis en $M$. ovinus y el potencial de $M$. ovinus para servir de vector ${ }^{16}$.

\section{Rickettsia}

M. ovinus está localizado principalmente en Europa, África noroccidental y Asia, causando inflamación, pérdida de lana, problemas cutáneos y transmisión de enfermedades. También funciona como vector. La presencia de M. ovinus, se observa en Rickettsia. En un estudio se recolectaron 95 ovejas keds en la región sur de la ciudad de Alaer, al noroeste de China. Se realizó PCR basado en seis fragmentos de genes de Rickettsia; (17-kDa) gen del antígeno, gen del ARNr 16S, gen del antígeno 4 de la célula de superficie, gen de la citrato sintasa y genes de proteínas A y B de la membrana externa. De acuerdo a la morfología y resultados del análisis molecular, la especie fue identificada como $M$. ovinus, con $100 \%$ de identidad de St. Kilda, Australia. Los marcadores genéticos de estos dos agentes rickettsiales amplificados en este estudio, mostraron diversidad molecular ${ }^{9}$.

\section{Bartonella melophagi}

Estudios actuales sugieren que B. melophagi puede estar asociado en bovinos, búfalos de agua, camellos, varias especies de ciervos y alces, pero en su mayoría existen en machos ovinos de Etiopía, siendo una comunidad que no ha reportado estudios o investigación de presencia de infecciones animales como humanas ${ }^{10}$ Debido a reportes realizados con pruebas PCR a 783 especímenes de Melophagus ovinus (ked de la oveja) recolectadas en ovejas adultas y jóvenes, arrojaron datos importantes en los cuales se teorizó que estos parásitos podrían estar conviviendo de una manera simbió- 
tica con $B$ melophagi, pero también se sugiere que las ovejas sean un huésped natural y la ked de la oveja sea su vector declarado ${ }^{6}$. Dentro de los signos clínicos en parasitosis por $M$. ovinus puede haber prurito, inflamación cutánea, caída de lana o pelo, miasis cutánea, disminución de la producción de lana, falta de desarrollo del animal y reducción de peso ?

\section{Theileria $s p$}

Es un parásito interno transmitido por garrapatas al ganado bovino y ovino, ocasionando pérdidas económicas, además que las especies de garrapatas miembros de Ixodidae y Argasidae de esta última incluyen 5 géneros y 200 especies, 118 de las cuales pertenecen al género Ornithodoros y se han reportado al menos 19 especies solo en China, ocasionando varias enfermedades y patologías en los ovinos, de las cuales la más importante mundialmente es la teileriosis ${ }^{17}$. En un estudio realizado en China de 38 secuencias de referencia analizadas y descartando las secuencias idénticas de Apicomplexa en muestras de Movinus de los condados de Kuqa, Aksu y Xinjiang, se arrojaron muestras positivas de Theileria ovis, que son frecuentemente encontradas en pequeños rumiantes ${ }^{17}$. De ellas, las cuatro secuencias obtenidas estaban todas asociadas con la rama de T. ovis. Así fue un primer estudio que informó la detección de ADN de T. ovis en $M$. ovinus y el primer estudio de identificación molecular para confirmar la detección de T. ovis en Ornithodoros lahorensis en China ${ }^{17}$. Otro estudio realizado en Ganze y Ngawa (China), donde se muestrearon yaks domésticos ( $\mathbf{n}=$ 144), ovejas tibetanas $(n=92)$ y caballos del Tíbet (n = 142), utilizando el ARNr 18S como gen de direccionamiento, se determinó una elevada tasa de infección y alta distribución geográfica de estos parásitos en la meseta tibetana oriental ${ }^{5}$. Así, las subespecies Theileria sinensis y T. luwenshuni fueron las especies dominantes que infectaron yaks y ovejas, respectivamente ${ }^{5}$. Las ked de las ovejas (M. ovinus) en el condado de Shiquse presentaron una prevalencia del T. hiwenshuni del $30,8 \%(8 / 26)$ en India ${ }^{5}$. Surge que su crecimiento, aunque no tiene un impacto muy grande, puede llegar a ser un problema zoonótico con el pasar del tiempo ${ }^{17}$.

\section{Virus de la lengua azul}

Se conoce que el principal agente parasitario que actúa como vector en la transmisión del virus de la lengua azul es el mosquito de la familia Culicoides ${ }^{11}$. Sin embargo, a lo largo de los años se han realizado estudios para determinar otros parásitos que puedan ay udar a su transmisión. En Canadá se realizaron una serie de experimentos para determinar si el Melophagus ovinus, que se alimentan de ovejas infectadas con el virus de la lengua azul, podían mantener el agente dentro de sus cuerpos e infectar a otros organismos susceptibles al ser inoculados por vía subcutánea. En los resultados se presentaron los mismos signos en los animales infectados por la inoculación que los que se presentaban por vía sanguínea, por lo que se sugirió un papel im- portante de este parásito en la transmisión del virus de la lengua azul ${ }^{4}$. En Denver, Colorado, se realizaron 5 estudios para demostrar que el Melophagus ovinus puede transmitir la enfermedad de la lengua azul. El primer experimento se llevó a cabo en una oveja susceptible al virus, que poseía 60 parásitos establecidos, a la cual se le inoculó el virus y luego fueron transferidos 5 agentes diariamente a otras ovejas susceptibles de 10 a 35 días, las cuales después fueron diagnosticadas con la enfermedad mediante pruebas de neutralización de suero. En el segundo experimento se colocaron 200 $k e d s$ en una oveja susceptible esplenectomizada y se le inoculó el virus. Desde el día 10 se transfirieron 25 agentes diariamente a cada una de dos ovejas susceptibles, hasta que se encontraron 100 keds en cada una, luego se extrajo la sangre de las dos y se les inoculó a otros dos animales. Todos presentaron signos de enfermedad. En el tercero, cuarto y quinto ensayo donde se traspasaron agentes y se inoculó el virus, no varió la aparición de animales positivos a los signos de la enfermedad de lengua azul. Este informe sugiere que la transmisión del agente es más de tipo mecánica que de tipo biológica ${ }^{10}$.

\section{Borrelia burgdorferi}

El agente bacteriano causante de la enfermedad de lyme es Borrelia burgdorferi sensu lato, cuyos vectores principales son las garrapatas del género ixodes. Sin embargo, un estudio realizado en Tíbet (China) determinó la presencia de B. burgdorferi en otros vectores. Se recolectaron al azar 1020 ectoparásitos de ovejas, de los cuales 1016 fueron $M$. ovinus, y se realizó un pool para efectuar un PCR que determinó la presencia de B. burgdorferi con una prevalencia de 3,6\%. A pesar de los resultados, el estudio tenía ciertas limitantes, ya que no se calcularon los ovinos muestreados, por lo tanto, no se determinó el número de ovinos con las moscas infectadas. Además, se debe recalcar que los ovinos no son hospedadores competentes, al igual que otro tipo de vectores ${ }^{3}$.

\section{CONCLUSIONES}

Se estableció la importancia de $M$. ovinus como vector de enfermedades en varios países del mundo, como Australia, China, India, Etiopia, Canadá, Estados Unidos, países del Medio Oriente y de América del Sur.

Se describió el efecto patógeno de M. ovinus dentro del ámbito veterinario, siendo el más afectado la producción de lana.

Se enumeraron los principales agentes patógenos de $M$. ovinus y sus efectos en la salud animal.

Agradecimientos. A nuestros padres y docentes por motivarnos en la formación académica, y por su ayuda a los estudiantes Mirella Simbaña, Carolina Soria, Daniela Serrano, Ximena Casco, Gabriel Chávez y Javier Roldán. 
tica con $B$ melophagi, pero también se sugiere que las ovejas sean un huésped natural y la ked de la oveja sea su vector declarado ${ }^{6}$. Dentro de los signos clínicos en parasitosis por $M$. ovinus puede haber prurito, inflamación cutánea, caída de lana o pelo, miasis cutánea, disminución de la producción de lana, falta de desarrollo del animal y reducción de peso ?

\section{Theileria $s p$}

Es un parásito interno transmitido por garrapatas al ganado bovino y ovino, ocasionando pérdidas económicas, además que las especies de garrapatas miembros de Ixodidae y Argasidae de esta última incluyen 5 géneros y 200 especies, 118 de las cuales pertenecen al género Ornithodoros y se han reportado al menos 19 especies solo en China, ocasionando varias enfermedades y patologías en los ovinos, de las cuales la más importante mundialmente es la teileriosis ${ }^{17}$. En un estudio realizado en China de 38 secuencias de referencia analizadas y descartando las secuencias idénticas de Apicomplexa en muestras de Movinus de los condados de Kuqa, Aksu y Xinjiang, se arrojaron muestras positivas de Theileria ovis, que son frecuentemente encontradas en pequeños rumiantes ${ }^{17}$. De ellas, las cuatro secuencias obtenidas estaban todas asociadas con la rama de T. ovis. Así fue un primer estudio que informó la detección de ADN de T. ovis en $M$. ovinus y el primer estudio de identificación molecular para confirmar la detección de T. ovis en Ornithodoros lahorensis en China ${ }^{17}$. Otro estudio realizado en Ganze y Ngawa (China), donde se muestrearon yaks domésticos ( $\mathbf{n}=$ 144), ovejas tibetanas $(n=92)$ y caballos del Tíbet (n = 142), utilizando el ARNr 18S como gen de direccionamiento, se determinó una elevada tasa de infección y alta distribución geográfica de estos parásitos en la meseta tibetana oriental ${ }^{5}$. Así, las subespecies Theileria sinensis y T. luwenshuni fueron las especies dominantes que infectaron yaks y ovejas, respectivamente ${ }^{5}$. Las ked de las ovejas (M. ovinus) en el condado de Shiquse presentaron una prevalencia del T. hiwenshuni del $30,8 \%(8 / 26)$ en India ${ }^{5}$. Surge que su crecimiento, aunque no tiene un impacto muy grande, puede llegar a ser un problema zoonótico con el pasar del tiempo ${ }^{17}$.

\section{Virus de la lengua azul}

Se conoce que el principal agente parasitario que actúa como vector en la transmisión del virus de la lengua azul es el mosquito de la familia Culicoides ${ }^{11}$. Sin embargo, a lo largo de los años se han realizado estudios para determinar otros parásitos que puedan ay udar a su transmisión. En Canadá se realizaron una serie de experimentos para determinar si el Melophagus ovinus, que se alimentan de ovejas infectadas con el virus de la lengua azul, podían mantener el agente dentro de sus cuerpos e infectar a otros organismos susceptibles al ser inoculados por vía subcutánea. En los resultados se presentaron los mismos signos en los animales infectados por la inoculación que los que se presentaban por vía sanguínea, por lo que se sugirió un papel im- portante de este parásito en la transmisión del virus de la lengua azul ${ }^{4}$. En Denver, Colorado, se realizaron 5 estudios para demostrar que el Melophagus ovinus puede transmitir la enfermedad de la lengua azul. El primer experimento se llevó a cabo en una oveja susceptible al virus, que poseía 60 parásitos establecidos, a la cual se le inoculó el virus y luego fueron transferidos 5 agentes diariamente a otras ovejas susceptibles de 10 a 35 días, las cuales después fueron diagnosticadas con la enfermedad mediante pruebas de neutralización de suero. En el segundo experimento se colocaron 200 $k e d s$ en una oveja susceptible esplenectomizada y se le inoculó el virus. Desde el día 10 se transfirieron 25 agentes diariamente a cada una de dos ovejas susceptibles, hasta que se encontraron 100 keds en cada una, luego se extrajo la sangre de las dos y se les inoculó a otros dos animales. Todos presentaron signos de enfermedad. En el tercero, cuarto y quinto ensayo donde se traspasaron agentes y se inoculó el virus, no varió la aparición de animales positivos a los signos de la enfermedad de lengua azul. Este informe sugiere que la transmisión del agente es más de tipo mecánica que de tipo biológica ${ }^{10}$.

\section{Borrelia burgdorferi}

El agente bacteriano causante de la enfermedad de lyme es Borrelia burgdorferi sensu lato, cuyos vectores principales son las garrapatas del género ixodes. Sin embargo, un estudio realizado en Tíbet (China) determinó la presencia de B. burgdorferi en otros vectores. Se recolectaron al azar 1020 ectoparásitos de ovejas, de los cuales 1016 fueron $M$. ovinus, y se realizó un pool para efectuar un PCR que determinó la presencia de B. burgdorferi con una prevalencia de 3,6\%. A pesar de los resultados, el estudio tenía ciertas limitantes, ya que no se calcularon los ovinos muestreados, por lo tanto, no se determinó el número de ovinos con las moscas infectadas. Además, se debe recalcar que los ovinos no son hospedadores competentes, al igual que otro tipo de vectores ${ }^{3}$.

\section{CONCLUSIONES}

Se estableció la importancia de $M$. ovinus como vector de enfermedades en varios países del mundo, como Australia, China, India, Etiopia, Canadá, Estados Unidos, países del Medio Oriente y de América del Sur.

Se describió el efecto patógeno de M. ovinus dentro del ámbito veterinario, siendo el más afectado la producción de lana.

Se enumeraron los principales agentes patógenos de $M$. ovinus y sus efectos en la salud animal.

Agradecimientos. A nuestros padres y docentes por motivarnos en la formación académica, y por su ayuda a los estudiantes Mirella Simbaña, Carolina Soria, Daniela Serrano, Ximena Casco, Gabriel Chávez y Javier Roldán. 


\section{REFERENCIAS}

1. Centro de sanidad animal. 2012. Melophagus ovinus (piojo o garrapata de la oveja), Internet: http://centrodesanidadanimal.blogspot.com/2012/2/melophagus-ovinus-pio jo-o-garrapata, html.

2. Centro de educación y tecnología. 2012. Manual control biológico de Melophagus ovinus. Internet: http://itas.cl/ wp/wp-content/uploads/2014/04/Manual-Melofago- CET. pdf

3. Chu CY et al. 2011. Borrelia burgdorferi sensu lato in sheep keds (Melophagus ovinus), Tibet, China. Internet: Veterinary Microbiology, Vol. 149, may 2011.

4. Gray DP, Bannister GL. 1961. Infectivity of the virus in the sheep ked, Melophagus ovinus. Studies on Bluetongue 25(9): 230-232.

5. Hao L et al. 2020 Detection of Theileria sp in ticks, sheep keds (Melophagus ovinus), and livestock in eastern tibetan plateau, China. Parasitology Res 1:4, 2020 ..

6. Kosoy M et al. 2016. Bartonella melophagi in blood of domestic sheep (Ovis aries) and sheep keds (Melophagus ovinus) from the southwestern US: cultures, genetic characterization, and ecological connections. Vet Microbiol 4: 7-24.

7. Kumsa B, Parola P, Raoult D, Socolovschi C. 2014. Bartonella melophagi in Melophagus ovinus (sheep ked) collected from sheep in northern Oromia, Ethiopia. Comp Immun Microbiol \& Infect Dis 4: 69-70.

8. Larroza M. 2013. Caracterización de la melofagosis en ovinos en la región patagónica: ciclo biológico, dinámica poblacional y distribución [Internet]. Univ. Nac. de la Plata, 2013. https://repositorio.inta.gob.ar.
9. Liu D et al. 2016 First report of Rickettsia raoultii and $R$. slovaca in Melophagus ovinus, the sheep ked. Parasit Vectors 1: 2016.

10. Luedke AJ, Jochim MM, Bowne JG. 1965. Preliminary bluetongue transmission with the sheep ked Melophagus ovinus. Can J Comp Med Vet Sci 29: 229-231.

11. OIE. 2020 (citado el 19 de agosto de 2020). Lengua azul [Internet]. Disponible en: www.oie.int/es/sanidad-animalen-el-mundo/fichas.

12. Olaechea F, Corley J, Larroza M, Cabrera FR. 2006. Ingreso y evolución del parasitismo por Melophagus ovinus en una majada Corriedale en el noroeste de la Patagonia Argentina. Parasitol Latinoam 61: 86-89.

13. Olaechea F, Larroza M, Raffo R, Cabrera F. 2013. Melophagus ovinus: infestación experimental de ovinos y supervivencia del parásito en el medio ambiente. Rev Med Vet (Bogotá) 88: 4, 158.

14. Olaechea FV. 2013. Aspectos básicos del ectoparásito más frecuente que afecta las majadas del sur patagónico, Melofagos ovinus. Veterinarios Patagónicos 2: 5, 5-7.

15. Thompson C, Godfrey S, Thompson A. 2014. Trypanosomes of Australian mammals: A review. Elsevier [Internet]. Disponible en: https://www.ncbi.nlm.nih.gov/ pmc/ articles $/ \mathrm{PMC} 4142263 / \mathrm{pdf} / \mathrm{main} . \mathrm{pdf} /$ ?tool=EBI

16. Zhao L et al. 2018. First report of Anaplasma ovis in pupal and adult Melophagus ovinus (sheep ked) collected in South Xinjiang, China. Parasit Vectors 20: 18.

17. Zhao L et al. 2019. Theileria ovis (Piroplasmida: Theileriidae) detected in Melophagus ovinus (Diptera: Hippoboscoidea) and Ornithodoros lahorensis (Ixodida: Argasidae) removed from sheep in Xinjiang, China. J Med Entomol 2019: 1-3. 